\title{
Parametric methods for gamma and inverse gamma correction, with extensions to halftoning
}

\author{
HAROLD STANISLAW \\ University of New South Wales, Kensington, New South Wales, Australia \\ and \\ LYNN A. OLZAK \\ University of California, Los Angeles, California
}

\begin{abstract}
Users of computer-controlled color video systems rely on procedures called gamma and inverse gamma correction to translate between system-specific descriptions of their stimuli and perceptually meaningful measures that are independent of the video system. These corrections are usually performed using a tabular approach. In the present paper, we detail problems with the tabular approach, suggest a parametric alternative, and describe programs for estimating parameter values. The parametric approach is then illustrated by using gamma and inverse gamma correction to find the best pair of colors for a halftone stimulus (i.e., one combining two different colors).
\end{abstract}

Computer-controlled color video systems can present dynamic stimuli, ramp static stimuli on and off, and respond to observer inputs by generating new stimuli in real time. Thus, they offer tremendous potential for studies of color vision (Vingrys \& King-Smith, 1986). However, psychologists cannot realize this potential without first solving a number of technical problems, one of which will be considered here.

Before this problem can be described, however, a review of video system operating technology will be in order. Color video systems generate images on cathoderay tube (CRT) monitors by manipulating the voltages that electron guns use to excite red, green, and blue phosphors on the CRT screen. In a computer-controlled system, a digital-to-analog converter (DAC) converts an integer gun setting to an electron gun voltage. The gun settings reside in the computer's color lookup table (LUT). Three gun settings-one each for the red, green, and blue guns-constitute an LUT entry. ${ }^{1}$ Colored images are generated by "painting" each pixel on the screen with a particular LUT entry, so that the LUT serves as the user's "palette.",

Colors on a CRT may be described by their corresponding LUT entries. However, the color produced by a given LUT entry is system-dependent and temporally unstable. Thus, it is desirable to describe colors in perceptually relevant terms, such as tristimulus coordinates in CIE

Portions of this work-which was supported in part by U. S. Coast Guard Contract DTCG-39-C-80205-were presented at the September, 1989, meeting of the Australian Psychological Society in Hobart, Tasmania. We thank David Brainard for providing us with calibration data, and Ghinwa Jalloul and two anonymous reviewers for making insightful comments on earlier versions of this manuscript. Requests for reprints should be sent to Harold Stanislaw, School of Psychology, University of New South Wales, P. O. Box 1, Kensington, NSW 2033, Australia.
$X Y Z$ color space (but see Ware \& Beatty, 1988, for an exception).

A description of this sort requires an understanding of two relationships-that between LUT entries and phosphor excitations, and that between phosphor excitations and tristimulus coordinates. The latter relationship is fixed and determined by the monitor's phosphors. The former relationship, called gamma correction, is more labile, varying with such factors as the system, time, and the monitor's contrast and intensity settings. Gamma correction is therefore determined empirically, by calibrating the monitor. Calibration methods have been discussed extensively elsewhere (Brainard, 1989; Cowan, 1983; Cowan \& Rowell, 1986) and will not be examined here. Instead, we will consider how the calibration data are used to convert LUT entries to excitations, and excitations to LUT entries.

We will begin by discussing current methods for performing gamma correction, and point out some difficulties with these methods. An alternative approach, and methods for implementing it, will then be described. We will conclude by applying the alternative approach to halftoning, which paints a stimulus with a combination of gun settings to achieve a desired color.

\section{Gamma Correction Methods}

The gamma function for an ideal video system is

$$
\frac{e}{e_{\max }}=\left(\frac{v}{v_{\max }}\right)^{\gamma}
$$

where $e$ is the excitation (e.g., in $\mathrm{cd} / \mathrm{m}^{2}$ ) of a given phosphor, $v$ is the gun voltage applied to that phosphor, $\gamma$ is a parameter that varies from gun to gun, and $e_{\max }$ is the excitation resulting from the maximum possible voltage, $v_{\max }$ (Cowan, 1983). The voltage produced by a given gun is ideally proportional to its gun setting, $s$. Further- 
more, $e_{\max }$ and $v_{\max }$ are constants, so that Equation 1 can be rewritten as

$$
e=k s^{\gamma}
$$

where $k$ is a constant.

Users are sometimes less interested in predicting excitations from gun settings than in determining the gun settings needed to present stimuli at a desired tristimulus coordinate (see Stanislaw, 1989). This requires inverse gamma correction. Rearrangement of Equation 2 yields the inverse gamma function for an ideal video system:

$$
s=\left(\frac{e}{k}\right)^{1 / \gamma}
$$

Unfortunately, Equations 2 and 3 are poor descriptors of actual systems. Previous authors (e.g., Mulligan, 1986; Watson et al., 1986) have therefore recommended using arrays of measured excitations, indexed by gun settings, to perform gamma correction. Similarly, arrays of gun settings, indexed by excitations, can be used for inverse gamma correction. This tabular approach is used widely, and it has been implemented directly on some systems (e.g., Macintosh II computers). However, there are several problems with it:

1. A complete table for a given gun can be generated only by measuring excitations at all possible gun settings. This is impractical, unless the measurement process is automated by feeding the output from a photometer directly into the computer through an analog-to-digital converter. The number of measurements can be reduced by sampling only a few gun settings, and using interpolation to complete the table. However, interpolation requires a mathematical model for the gamma function, which is precisely what the tabular method attempts to avoid.

2. Gamma functions should increase monotonically; larger gun settings should produce greater excitations. However, tabular data may be nonmonotonic, due to sampling and/or measurement error. This problem can be corrected by smoothing the data, but smoothing again requires the use of a mathematical model.

3. Tables for inverse gamma correction can provide access to all possible gun settings only if they contain far more entries than there are possible settings. This results from the nonlinear nature of the inverse gamma function, and from roundoff error that is introduced when (fractional) excitations are converted to (integer) array indices.

4. The tabular method can be applied only if the gamma function for each gun is unaffected by the settings for the remaining guns. This property of gun independence can often be obtained by adjusting the CRT appropriately (Cowan, 1983). However, in its absence, the gamma correction table must use all three gun settings as indexing variables. This is unfeasible: A system incorporating 8-bit DACs would require a gamma table with $2^{24}$ entries.

These problems are avoided by adopting a parametric approach for gamma and inverse gamma correction. In our experience, gamma correction is described to a high degree of accuracy by the equation $e= \begin{cases}\exp \left[p_{0}+p_{1}(\ln s)+p_{2}(\ln s)^{2}+p_{3}(\ln s)^{3}\right] & \text { if } s>s_{0} \\ q_{0}+q_{1} s+q_{2} s^{2} & \text { if } s \leq s_{0}\end{cases}$

where $p_{0}$ to $p_{3}$ are parameters of a power function, $q_{0}$ to $q_{2}$ are parameters of a quadratic function, and $s_{0}$ marks the transition from the power to the quadratic function. This equation is based on Cowan's (1983) gamma function, in which the logarithm of excitation is a quadratic function of the logarithm of the gun setting. However, Cowan's function applies to only a limited range of gun settings. Our experience suggests that an additional (i.e., cubic) term is needed to describe the gamma function over all possible settings. Furthermore, the power function works poorly at low gun settings, where a quadratic function provides a better fit.

Equation 4 describes the gamma function for a particular gun of interest, and assumes gun independence. This assumption can be relaxed by adding terms that involve the settings of the two guns that are not of interest. Thus, in the absence of gun independence, the function for predicting the excitation of red phosphors might include the green and blue gun settings, as well as the red gun setting. However, we do not pursue this possibility, as the terms that must be added to correct for nonindependence are likely to vary with the system.

The color resulting from a particular LUT entry can be described in terms of $X Y Z$ coordinates by using Equation 4 to convert LUT entries to excitations. The excitations can then be converted to $X Y Z$ coordinates using the equation

$$
[X Y Z]=\left[N_{\mathrm{r}} N_{\mathrm{g}} N_{\mathrm{b}}\right]\left[e_{\mathrm{r}} e_{\mathrm{g}} e_{\mathrm{b}}\right]\left[\begin{array}{ccc}
x_{\mathrm{r}} & y_{\mathrm{r}} & z_{\mathrm{r}} \\
x_{\mathrm{g}} & y_{\mathrm{g}} & z_{\mathrm{g}} \\
x_{\mathrm{b}} & y_{\mathrm{b}} & z_{\mathrm{b}}
\end{array}\right],
$$

where the subscripts denote the phosphor color (red, green, or blue), the $N$ s are normalizing factors, and the entries in the rightmost matrix describe the chromaticity coordinates of the CRT phosphors. Cowan (1983) describes how the normalizing factors can be found. The chromaticity coordinates should ideally be measured using a spectrophotometer (see Brainard, 1989, for details), but they may also be obtained from the monitor's manufacturer. The nominal operating characteristics of commonly used phosphors are described in some texts (e.g., Say, Hedler, Maninger, Momberger, \& Robbins, 1986), while a complete list for all registered phosphors is available from the Electronic Industries Association (1980). ${ }^{2}$ However, Cowan (1983) cautions against using nominal values.

The LUT entry needed to produce a color with a particular $X Y Z$ coordinate can be found by using the inverse of Equation 5 ,

$$
\left[e_{\mathrm{r}} e_{\mathrm{g}} e_{\mathrm{b}}\right]=\left[N_{\mathrm{r}} N_{\mathrm{g}} N_{\mathrm{b}}\right]^{-1}[X Y Z]\left[\begin{array}{ccc}
x_{\mathrm{r}} & y_{\mathrm{r}} & z_{\mathrm{r}} \\
x_{\mathrm{g}} & y_{\mathrm{g}} & z_{\mathrm{g}} \\
x_{\mathrm{b}} & y_{\mathrm{b}} & z_{\mathrm{b}}
\end{array}\right]^{-1},
$$

to determine the requisite excitations. Inverse gamma correction can then be performed by finding the roots of 
Equation 4, which indicate the gun settings needed to produce the requisite excitations.

The parameters in Equation 4 and the normalizing factors in Equations 5 and 6 vary with the monitor's contrast and intensity settings, and they may drift over time. Thus, they must be reestimated whenever the monitor is recalibrated (e.g., following adjustment of the CRT controls). RGBXYZ and XYZRGB are published FORTRAN routines that illustrate how Equations 4, 5, and 6 can be implemented to convert LUT entries to $X Y Z$ values, and $X Y Z$ values to LUT entries, respectively (Stanislaw, 1989).

\section{Constraints on Parameter Values}

The parametric approach requires that several constraints be satisfied:

Intersection. Both functions should predict the same excitation, $e_{0}$, at $s=s_{0}$. This will be the case if

$$
\begin{aligned}
p_{0}= & \ln \left(q_{0}+q_{1} s_{0}+q_{2} s_{0}^{2}\right)-p_{1}\left(\ln s_{0}\right) \\
& -p_{2}\left(\ln s_{0}\right)^{2}-p_{3}\left(\ln s_{0}\right)^{3} .
\end{aligned}
$$

Real numbers. To avoid taking the logarithm of a nonpositive number in Equation 4, $s_{0}$ must be greater than 0 . Similarly, the constraint

$$
q_{0}+q_{1} s_{0}+q_{2} s_{0}^{2}>0
$$

must be met to avoid taking the logarithm of a nonpositive number in Equation 7.

Monotonicity. Both functions should predict an increase in $e$ with an increase in $s$. The power function is monotonic if the zero crossings of its second derivative,

$$
\exp \left(\frac{2 p_{2} \pm \sqrt{p_{2}^{2}-3 p_{1} p_{3}}}{3 p_{3}}\right),
$$

fall outside the interval $\left\{s_{0}, s_{\max }\right\}$, where $s_{\max }$ is the maximum possible gun setting (e.g., 255 in a system with an 8-bit DAC). Our experience suggests there is no need to test whether the function is actually increasing over this interval; any reasonable set of parameter estimates yields an increasing function.

The quadratic function is monotonic increasing whenever it has a nonnegative first derivative at both 0 and $s_{0}$. This obtains if

$$
q_{1} \geq 0
$$

and

$$
q_{1}+2 q_{2} s_{0} \geq 0 .
$$

An additional constraint that might be imposed is for the first derivatives of the two functions to be equal at $s_{0}$, providing a smooth transition from one function to the other. However, in actual practice, the two functions will "meet" only in the rare instance when $s_{0}$ is an integer (i.e., a valid gun setting). ${ }^{3}$ Furthermore, a smoothness constraint makes parameter estimation overly sensitive to the initial parameter estimates. Thus, we see no need for a smoothness constraint. In fact, the first derivatives of the two functions are usually quite similar at $s_{0}$, even in the absence of a smoothness constraint.

\section{Parameter Estimation}

Least-squares estimates of the gamma function parameters can be found by minimizing the sum of the squared deviations between the measured excitations and those predicted from Equation 4. However, the partial derivative of $s_{0}$ with respect to the residual sum of squares, $S S_{\text {resid, }}$, is undefined. Thus, we estimate parameters using Powell's conjugate method, which does not require derivatives.

The conjugate method requires initial parameter estimates, which are then modified in an iterative manner to obtain final values. The initial estimates should satisfy the constraints outlined above and be reasonably close to the final values; otherwise, the conjugate method may fail to converge, or it will converge upon a local, rather than global, minimum.

If a value for $s_{0}$ is assumed a priori, initial parameter estimates for the power function can be found by applying multiple regression to its linear equivalent,

$$
\begin{aligned}
\ln (e)= & p_{0}+p_{1}(\ln s)+p_{2}(\ln s)^{2} \\
& +p_{3}(\ln s)^{3} \text { for all } s>s_{0} .
\end{aligned}
$$

These parameters can be used to estimate $e_{0}$, by setting $s=s_{0}$. Multiple regression can then be used to estimate initial parameters for the quadratic function from the equation

$$
e^{\prime}=q_{1} s^{\prime}+q_{2}\left(s^{\prime}\right)^{2} \text { for all } s \leq s_{0},
$$

where $e^{\prime}=e-e_{0}$, and $s^{\prime}=s-s_{0}$.

Equation 13 lacks an intercept. The intercept for the quadratic function, $q_{0}$, should be determined as

$$
q_{0}=e_{0}-q_{1} s_{0}-q_{2} s_{0}^{2},
$$

so that the quadratic and power functions intersect at $s_{0}$.

This procedure is biased, in that parameters for the power function are estimated before those for the quadratic function. Note also that the multiple regression solution of Equation 12 minimizes errors in predicting the logarithms of the excitations, rather than the excitations themselves. Thus, the estimates produced by this method are only approximate.

The method is implemented in FORTRAN program GAMMA0, which proceeds as follows for each gun:

1. Calibration data are read from file GAMMA.DAT, which contains gun settings and the corresponding excitations (see Table 1).

2 . The calibration data are sorted in order of increasing gun setting, for reasons discussed below.

3. Squared gun settings, and the logarithms, squared logarithms, and cubed logarithms of all nonzero gun settings are determined.

4. The values of $s_{0}$ and $S S_{\text {resid }}$ are initialized to 0.5 and $10^{98}$, respectively. 
Table 1

\begin{tabular}{|c|c|c|c|c|c|c|}
\hline \multirow{3}{*}{$\begin{array}{l}\text { Gun } \\
\text { Setting }\end{array}$} & \multicolumn{6}{|c|}{$\begin{array}{l}\text { Calibration Data and Prediction Errors for a Number Nine } \\
\text { Graphics } 232808 \text { Board, Driving a Barco } 5135 \text { Monitor }\end{array}$} \\
\hline & \multicolumn{3}{|c|}{ Measured Relative Excitation } & \multicolumn{3}{|c|}{ Actual Minus Predicted Excitation } \\
\hline & Red & Green & Blue & Red & Green & Blue \\
\hline 0 & .002071 & .001201 & .001327 & -.000005 & .000016 & -.000207 \\
\hline 10 & .003089 & .001661 & & 99 & & .000242 \\
\hline 20 & .005702 & .003167 & .006 & .000006 & -.000074 & .000234 \\
\hline 30 & .010355 & .006424 & .011 & .000097 & -.000442 & -.000288 \\
\hline 40 & .017496 & & & -.000051 & -.000978 & -.000240 \\
\hline 50 & .027301 & & & .000034 & -.000976 & .000257 \\
\hline 60 & .039641 & & & .000011 & -.001136 & -.000065 \\
\hline 70 & .055288 & .047842 & .0604 & .000460 & .000850 & .000120 \\
\hline 80 & .073551 & .064010 & .0799 & .000514 & -.000280 & .000097 \\
\hline 90 & .094903 & .084319 & .1018 & .000489 & -.000561 & -.000493 \\
\hline 100 & .119592 & .108869 & .1285 & .000486 & -.000049 & .000486 \\
\hline 110 & .147163 & .136375 & .1565 & -.000084 & -.000172 & -.000528 \\
\hline 120 & .179388 & .167609 & .1895 & .000429 & -.000285 & .000118 \\
\hline 130 & .214539 & 203959 & .2258 & .000184 & .000885 & .000586 \\
\hline 140 & .253236 & & & -.000302 & .000018 & -.000729 \\
\hline 150 & .296986 & .286555 & .3079 & .000381 & .001 & .000329 \\
\hline 160 & .342996 & .334010 & .354331 & -.000646 & .001395 & -.000019 \\
\hline 170 & .394060 & .384010 & .403227 & -.000672 & -.000072 & -.001684 \\
\hline 180 & .449911 & .441877 & .459390 & -.000038 & .002061 & .000019 \\
\hline 190 & .507979 & .498715 & .520392 & -.001382 & -.001164 & .002578 \\
\hline 200 & .573582 & .562982 & .580814 & .000551 & -.001348 & .000496 \\
\hline 210 & .641179 & .632391 & .648256 & .000161 & -.000829 & .001293 \\
\hline 220 & .711348 & .704370 & .715116 & -.002025 & -.002226 & -.002709 \\
\hline 230 & .792287 & .784062 & .790698 & .002141 & -.000437 & -.002282 \\
\hline 240 & .873227 & .868895 & .875000 & .001846 & .001929 & .002497 \\
\hline 250 & .957447 & .953728 & .956395 & .000329 & -.000302 & -.000073 \\
\hline
\end{tabular}

Note-See Brainard, 1989, for details. The first four columns of the table constitute file GAMMA.DAT; they contain gun settings and the excitations that result when these settings are applied to the red, green, and blue guns. Excitations were predicted using the final parameter values listed in Table 2

5. The quantities needed for the multiple regression solution of Equation 12-including the sums of squares and crossproducts for the logarithms of the excitations, the logarithms of the gun settings, the squared logarithms of the gun settings, and so on-are found.

6 . The sums of squares and crossproducts are used to determine the intercorrelations among all variables in Equation 12 (the logarithm of the excitation, and the logarithm of the gun setting raised to the first, second, and third powers).

7. The correlation matrix is inverted to obtain multiple regression estimates of the values for parameters $p_{0}$ to $p_{3}$ in Equation 12.

8. The residual sum of squares for the power function, $S S_{\text {power }}$, is calculated.

9. The value for $e_{0}$ is calculated from the power function by setting $s=s_{0}$.

10. The number of gun settings belonging to the quadratic function, $n_{\text {quad }}$, is found. If $n_{\text {quad }}>0$, GAMMA0 determines values for the parameters of the quadratic function, and uses these to calculate the residual sum of squares for the quadratic function, $S S_{\text {quad. }}$. Otherwise, $S S_{\text {quad }}$ is set to 0 .

Parameters for the quadratic function are estimated using one of two approaches. If $n_{\mathrm{quad}}=1, q_{2}$ is set to zero, and $q_{1}$ is set so that the quadratic function passes through the lone point in the quadratic function and predicts an excitation of $e_{0}$ at $s_{0}$. If $n_{\text {quad }}>1, q_{1}$ and $q_{2}$ are estimated using multiple regression. Thus, $e^{\prime}$ and $s^{\prime}$ are found for all gun settings in the quadratic function, the correlations between $e^{\prime}, s^{\prime}$, and $\left(s^{\prime}\right)^{2}$ are found by calculating the sums of their squares and crossproducts, and the correlation matrix is inverted. For both approaches, $q_{0}$ is set according to Equation 14.

11. $S S_{\text {resid }}$ is found by summing $S S_{\text {power }}$ and $S S_{\text {quad. If }}$ this is less than the previous best value of $S S_{\text {resid, and if }}$ the monotonicity constraints are satisfied, the current parameter estimates are retained; otherwise, they are discarded. The intersection and real number constraints are not tested, for the estimation procedure guarantees they will be satisfied.

12. The value of $s_{0}$ is incremented by 0.5 . If $s_{0}$ now exceeds $s_{\max }$, GAMMA0 proceeds to Step 13. Otherwise, GAMMA0 determines whether, with the new value of $s_{0}$, a point has migrated from the power to the quadratic function. If it has, the program adjusts the sums of squares and crossproducts accordingly, and returns to Step 6. If no point has migrated, the program returns to Step 9.

13. GAMMA0 writes the final parameter estimates and $S S_{\text {resid }}$ to file GAMMA.R (for the red gun), GAMMA.G (for green), or GAMMA.B (for blue).

GAMMA0 is written in double precision, because single precision is inadequate. Considerable computational economy results from sorting the data in order of increas- 
ing gun setting. This allows the program to simply adjust the sums and crossproducts for points that migrate from one function to the other in Step 12, rather than return to Step 5 and calculate the quantities anew for the entire data set.

FORTRAN program GAMMA uses the conjugate method to convert the initial parameter estimates to final values. GAMMA is written in double precision and proceeds as follows for each gun:

1. Calibration data are read from file GAMMA.DAT (see Table 1).

2. Initial estimates for all parameters except $p_{0}$ (which is determined in Step 6 below) are obtained from file GAMMA.R, GAMMA.G, or GAMMA.B. The parameter estimates in these files are ordinarily provided by GAMMA0. However, exceptions may occur if the system has been calibrated previously (i.e., GAMMA has already been run), and if the monitor contrast and intensity settings have not been changed. The final parameter values from the previous calibration (saved in Step 7 below) may then serve as initial estimates for the present calibration.

3 . The calibration data are sorted in order of increasing gun setting, to reduce the time needed to calculate

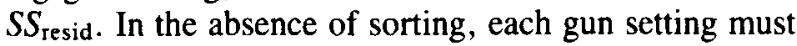
be compared to $s_{0}$ to determine whether the excitation should be predicted using the power or quadratic function. However, if the data are sorted, there is no need to compare the gun setting to $s_{0}$ once a setting that exceeds $s_{0}$ is encountered; all subsequent excitations should be predicted using the power function.

4. Squared gun settings, and the logarithms, squared logarithms, and cubed logarithms of all nonzero gun settings are determined. This again reduces the time needed to find $S S_{\text {resid, }}$, because the quantities are calculated only once, instead of each time $S S_{\text {resid }}$ must be found.

5 . The parameter values that minimize $S S_{\text {resid }}$ are determined in an iterative manner using the published subroutines POWELL, LINMIN, BRENT, and MNBRAK (Press, Flannery, Teukolsky, \& Vetterling, 1986, pp. 294-301), which implement Powell's conjugate method. For optimization purposes, POWELL is inserted directly within GAMMA, and BRENT and MNBRAK are inserted directly within LINMIN. This reduces execution time by eliminating subroutine calls. The calls that remain (e.g., to LINMIN) pass arguments through COMMON statements, which saves time by reducing manipulation of the stack. The iterations continue for 200 cycles, or until convergence is achieved $\left(S S_{\text {resid }}\right.$ changes by less than $10^{-6}$ on successive iterations).

The routines published by Press et al. (1986, pp. 294301) minimize the value returned by a function they call FUNC. In GAMMA, FUNC returns $S S_{\text {resid, }}$ using Equation 4 to generate predicted excitations. FUNC also tests the parameter constraints, setting $S S_{\text {resid }}$ to an extremely large number $\left(10^{98}\right)$ if they are violated. This is preferable to rejecting the parameter estimates directly if they violate the constraints, since the latter approach may cause the conjugate method to fail.
To reduce execution time, GAMMA calls FUNC directly, rather than through function FIDIM (as suggested by Press et al., 1986, p. 300). FUNC should be highly optimized, since it may be called several thousand times to estimate a single set of parameters. This accounts for the sorting in Step 3, and for our reluctance to verify that the power function increases over the interval $\left\{s_{0}, s_{\max }\right\}$.

6 . The value of $p_{0}$ is determined from the final parameter estimates, using Equation 7 . The final value for $S S_{\text {resid }}$ is also found.

7. Final parameter values and goodness of fit statistics (deviations between predicted and actual excitations and the squared correlation between these values) are output, overwriting the original input file (GAMMA.R, GAMMA.G, or GAMMA.B).

GAMMA and GAMMA0 differ in the method used to satisfy the intersection constraint. GAMMA estimates all parameters except $p_{0}$, which is set using Equation 7 . GAMMA0 estimates all parameters except $q_{0}$, which is set using Equation 14. Both approaches are valid; the intersection constraint simply implies that one parameter is fixed, given all other parameters. The approach adopted in each program is a matter of convenience.

\section{Goodness of Fit}

Equation 4 was developed for a Barco 5137 monitor, driven by a CAT 1631 graphics board. It is therefore no surprise that the equation works well for this system. A more stringent test of the equation is its ability to perform gamma correction for other systems. Thus, we report on the equation's goodness of fit for the data in Table 1, which apply to a different system (detailed in Brainard, 1989) and were kindly provided to us by David Brainard of Stanford University.

Table 2 lists the parameter estimates produced for these data by GAMMAO and GAMMA. The estimates were

Table 2

Initial and Final Parameter Estimates Produced by GAMMA0 and GAMMA (respectively), for the Data in Table 1

\begin{tabular}{clrrr}
\hline \multirow{4}{*}{ Parameter } & $\begin{array}{c}\text { Type of } \\
\text { Estimate }\end{array}$ & \multicolumn{1}{c}{ Red Gun } & Green Gun & \multicolumn{1}{c}{ Blue Gun } \\
\hline$q_{0}$ & Initial & 0.002070 & 0.001193 & 0.001523 \\
& Final & 0.002077 & 0.001185 & 0.001534 \\
$q_{1}$ & Initial & 0.000024 & 0.000002 & 0.000000 \\
& Final & 0.000023 & 0.000002 & 0.000000 \\
$q_{2}$ & Initial & 0.000008 & 0.000005 & 0.000012 \\
& Final & 0.000008 & 0.000005 & 0.000012 \\
$s_{0}$ & Initial & 26.000000 & 25.000000 & 53.000000 \\
& Final & 26.424615 & 23.070809 & 53.011127 \\
$p_{0}$ & Initial & -6.246694 & -10.324249 & -10.245431 \\
& Final & -6.258586 & -9.682451 & -10.244416 \\
$p_{1}$ & Initial & -1.280172 & 0.453529 & 1.382027 \\
& Final & -1.278389 & 0.242092 & 1.381860 \\
$p_{2}$ & Initial & 0.658275 & 0.428575 & 0.095068 \\
& Final & 0.658321 & 0.436340 & 0.095066 \\
$p_{3}$ & Initial & -0.040384 & -0.031463 & -0.001949 \\
& Final & -0.040377 & -0.029726 & -0.001950 \\
\hline
\end{tabular}

Note-The estimates produced by GAMMA0 were used as initial estimates by GAMMA. 
produced in less than $1 \mathrm{~min}$ on a $16-\mathrm{MHz}$ computer equipped with a math coprocessor and an optimizing compiler. The accuracy of the gamma functions is evident from the small deviations between the predicted and actual relative excitations (Table 1). In fact, the squared correlation between these excitations exceeds 0.9999 for all three guns. Thus, the gamma functions account for over $99.99 \%$ of the variance in relative excitation. Clearly, the parametric approach works well for this system. Similar results apply to the Barco/CAT system for which the approach was developed.

\section{Inverse Gamma Correction}

Inverse gamma correction involves finding the gun setting needed to produce a desired excitation, $e_{\text {desire. }}$. This setting is related to the roots of the equation

$0=\left\{\begin{aligned} p_{0}-\ln \left(e_{\text {desire }}\right)+p_{1}(\ln s)+ & p_{2}(\ln s)^{2}+p_{3}(\ln s)^{3} \\ & \text { if } e_{\text {desire }}>e_{0} \\ q_{0}-e_{\text {desire }}+q_{1} s+q_{2} s^{2} & \text { if } e_{\text {desire }} \leq e_{0}\end{aligned}\right.$

Press et al. (1986, pp. 145-146) describe methods for finding these roots with a minimum of roundoff error. When $e_{\text {desire }} \leq e_{0}$, the gun setting is the root itself. Two roots will exist, but only one of these will fall within the interval $\left\{0, s_{0}\right\}$. When $e_{\text {desire }}>e_{0}$, the gun setting is the antilogarithm of the root. One or three real roots may result from this equation; the correct root will yield a gun setting in the interval $\left\{s_{0}, s_{\max }\right\}$.

\section{Halftoning}

Gun settings are restricted to integer values, which limits the number of colors that a video system can actually generate. This may create problems for applications that demand precise color control: When the gun setting returned by the inverse gamma function is rounded off to produce a valid (i.e., integer) value, the resulting excitation may deviate significantly from what is desired, resulting in quantization error.

Mulligan (1986) has shown how quantization errors can be reduced by halftoning (also known as "dithering"). Readers not familiar with halftoning are referred to Mulligan's treatment of the subject; here, we demonstrate how the parametric approach to gamma and inverse gamma correction can be used in halftoning applications.

Halftoning simulates noninteger gun settings by intermixing pixels with different integer gun settings, and ensuring that stimuli are viewed at a distance that prevents observers from resolving the individual pixels. For example, suppose the inverse gamma function prescribes a gun setting of 245.5 . This may be simulated by painting half the stimulus pixels with a gun setting of 245 , and half with a gun setting of 246 . The resulting stimulus will then have an excitation midway between the excitations produced by gun settings of 245 and 246 . This is not the same as applying a gun setting of 245.5 ; halftoning averages excitations, not gun settings. However, the quantization error will be smaller than it would be if a single gun setting were used for all pixels.

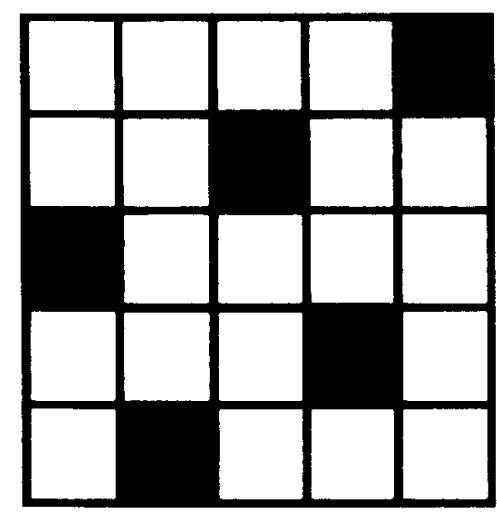

Figure 1. A 25-pixel square, in which $80 \%$ of the pixels are painted with a gun setting of 218 (indicated by empty cells), and $20 \%$ with a gun setting of 219 (indicated by shaded cells).

Halftoning offers considerable flexibility, since gun settings may be combined in unequal proportions and differ by more than 1 . For example, suppose a user of the video system described in Table 1 required a relative excitation of 0.7 for the red pixels. Using inverse gamma correction and the final parameter values listed in Table 2, this corresponds to a red gun setting of 218.2. However, since 218.2 is not an integer, this gun setting cannot actually be used. The two nearest settings that can be used are 218 and 219 , which (according to the final parameter values listed in Table 2) produce relative excitations of 0.698550 and 0.705940 , respectively. The excitation produced by 218 is the better of the two; a stimulus painted entirely with this setting would have a quantization error of 0.001450 , or about $0.2 \%$.

If this error is deemed excessive, halftoning can be used to reduce it further. One possibility is to paint $80 \%$ of the pixels with a gun setting of 218 , and the remainder with a gun setting of 219 , as illustrated in Figure 1. The quantization error resulting from a mixture of $n_{1}$ pixels with an excitation of $e_{1}$, and $n_{2}$ pixels with an excitation of $e_{2}$, is

$$
\left|e_{\text {desire }}-\frac{n_{1} e_{1}+n_{2} e_{2}}{n_{1}+n_{2}}\right|
$$

Thus, the quantization error in the present example is $0.000028(0.004 \%)$.

When pixels are mixed in unequal proportions, the size of the stimulus is constrained; the 4:1 ratio required for the present example can only be realized in a stimulus composed of an integral number of 5-pixel units. Furthermore, the stimulus may not be chromatically homogeneous: Since only 1 pixel in 5 is painted with a gun setting of 219 , some of the observer's receptive fields may be stimulated only by pixels set to 218 , while others may be stimulated by pixels set to both 218 and 219 . If these problems are considered serious, an alternate possibility is to search for two gun settings that produce the desired excitation when combined in equal proportions.

The double-precision FORTRAN program HALFTN conducts such a search as follows: 


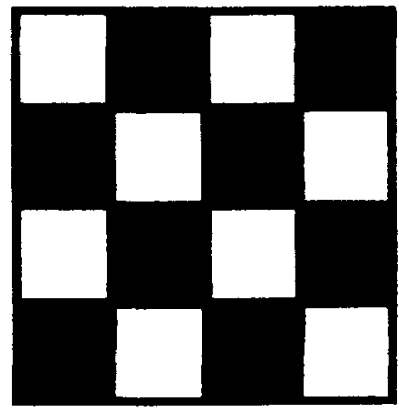

Figure 2. A 16-pixel square, painted with gun settings of 197 (empty cells) and 237 (shaded cells) in equal proportion.

1. Parameter values for the gun of interest are read from file GAMMA.R, GAMMA.G, or GAMMA.B.

2. The minimum quantization error is initialized at $10^{98}$, and one of the two gun settings for the halftoned stimulus, $s_{1}$, is initialized at 0 .

3. Equation 4 (i.e., gamma correction) is used to determine the excitation, $e_{1}$, resulting from $s_{1}$.

4. As is evident from Expression 16, if half the pixels in a stimulus have an excitation of $e_{1}$, quantization error will be minimized by setting the excitation $e$ of the remaining pixels to

$$
e=2 e_{\text {desire }}-e_{1} \text {. }
$$

HALFTN uses the roots of Equation 15 (i.e., inverse gamma correction) to determine the gun setting, $s$, that corresponds to $e$ in Equation 17.

5 . Since $s$ is unlikely to be an integer, the effects of rounding $s$ up are considered. Thus, Equation 4 (gamma correction) is used to determine the excitation $e_{2}$ that results from a gun setting of $s_{2}$, where $s_{2}$ is the minimum of int $(s+1)$ and $s_{\max }$. If the quantization error is less than the previous minimum, $s_{1}$ and $s_{2}$ are retained as the best gun settings found so far; otherwise, they are discarded. Similarly, the effects of rounding $s$ down are considered by using Equation 4 to find $e_{2}$ when $s_{2}$ is the maximum of int $(s)$ and 0 . Again, $s_{1}$ and $s_{2}$ are retained if the quantization error is less than the previous minimum; otherwise, they are discarded.

6. $s_{1}$ is incremented. If $s_{1}$ does not exceed $s_{\max }$, HALFTN returns to Step 3. Otherwise, HALFTN returns the best pair of gun settings that was found, as well as the corresponding quantization error.

HALFTN reveals that, in order to produce a relative excitation of 0.7 for the red pixels, gun settings of 197 and 237 should be combined in equal proportions. A checkerboard scheme can be used to paint a stimulus with these two settings (see Figure 2), which results in a quantization error of $0.000010(0.0014 \%)$. Smaller errors can be achieved by painting the stimulus with three or more gun settings. HALFTN could be expanded to accommodate this possibility, but we leave this exercise to the interested reader.

\section{Program Availability}

The source code for the programs described above may be obtained by sending a DOS-formatted 5.25 -in. disk, or a DOS- or Macintosh-formatted 3.5-in. disk, to the first author. The programs can also be obtained via electronic mail from the second author, at olzak@cognet.ucla.edu.

\section{REFERENCES}

Brainard, D. H. (1989). Calibration of a computer controlled color monitor. Color Research \& Application, 14, 23-34.

CowaN, W. B. (1983). An inexpensive scheme for calibration of a colour monitor in terms of CIE standard coordinates. Computer Graphics, 17, 315-321.

CoWAN, W. B., \& Rowell, N. (1986). On the gun independence and phosphor constancy of colour video monitors. Color Research \& Application, 11, S34-\$38.

ELECTRONIC INDUSTRIES AsSoCIATION. (1980). Optical characteristics of cathode ray tube screens (Tube Engineering Panel Advisory Council Publication No. 116). Washington, DC: Author.

Mulligan, J. B. (1986). Minimizing quantization errors in digitallycontrolled CRT displays. Color Research \& Application, 11, S47-S51.

Press, W. H., Flannery, B. P., Teukolsky, S. A., \& VetterLING, W. T. (1986). Numerical recipes: The art of scientific computing. Cambridge, England: Cambridge University Press.

Say, D. L., Hedler, R. A., Maninger, L. L., Momberger, R. A., \& RobBins, J. D. (1986). Monochrome and color image-display devices. In K. B. Benson (Ed.), Television engineering handbook (pp. 12.1-12.53). New York: McGraw-Hill.

Stanislaw, H. (1989). Lookup tables for linear trajectories through color space. Behavior Research Methods, Instruments, \& Computers, 21, 502-524.

VINGRYs, A. J., \& King-Smith, P. E. (1986). Factors in using color video monitors for assessment of visual thresholds. Color Research \& Application, 11, S57-S62.

WARE, C., \& BeatTY, J. C. (1988). Using color dimensions to display data dimensions. Human Factors, 30, 127-142.

Watson, A. B., Nielsen, K. R. K., Poirson, A., Fitzhugh, A., Bilson, A., Nguyen, K., \& Ahumada, A. J., JR. (1986). Use of a raster framebuffer in vision research. Behavior Research Methods, Instruments, \& Computers, 18, 587.594.

\section{NOTES}

1. The term LUT entry is sometimes applied to both the integer value used to set the voltage of a given gun and the trivariate assemblage of these values for all three guns. To avoid confusion, we refer to the former as gun settings, and to the latter as LUT entries.

2. Phosphors are identified by a code that begins with the letter $P$, which is followed by a number; many monitors use P22 phosphors. Publications generally list only the $x$ - and $y$-coordinates, but $z$ is readily determined using the equation $z=1-x-y$.

3. The two functions should nevertheless predict identical excitations at $s_{0}$, because this will ensure that the gamma function is monotonic across the entire range of gun settings.

(Manuscript received January 25, 1990; revision accepted for publication June 15, 1990.) 